\title{
Investigations in high speed blanking: cutting forces and microscopic observations
}

\author{
C. Gaudillière ${ }^{1, a}$, N. Ranc ${ }^{1}$, A. Larue ${ }^{1}$, and P. Lorong ${ }^{1}$ \\ PIMM, Arts et Métiers Paris Tech, 75013 Paris, France
}

\begin{abstract}
A new hopefull technique, called high speed blanking, has been investigated since few years. To understand the cutting process and how the tools have to be designed, this study is interrested in the cutting force measurement. A new cutting force measurement device has to be designed consider the industrial interest of such a study. The designed test bench induces a calibration process in order to stucy the cutting forces evolution. The paper is discussing the result that the peack load seems to decrease when the punch speed increases. Finally microscopic observations are made in order to find Adiabatic Shear Bands.
\end{abstract}

\section{Introduction}

Since around fifty years, no fundamental innovation has been developed in the sheet metal blanking field [1]. Recently, a new machining process has been investigated: the high speed blanking involving high cutting speeds, around $10 \mathrm{~m} / \mathrm{s}$. This new hopeful technique seems to be cheaper than fine blanking because this technique involves the use of cheaper tools without draw bead or very hard specifications. Jana and al. showed that generally the amount of doming (which corresponds of the flatness of the sheet metal after cutting) is reduced by using high punch speed [2]. However the speed seems to have no influence on edge taper which evaluates the perpendicular default. So, dimensional and geometrical workpiece qualities seem to be better than those obtained with classical blanking, but worse than those obtained with fine blanking. Finally this technique does not need lubricants, which avoids the use of degreasing machines [1].

Nevertheless, the inherent hard dynamic of such a process makes difficult the development of a high speed punch press. To avoid dies breaks, the design of the punch has to be adjusted. The fracture phenomenon governing the cutting process has to be well understood in order to design the machine bed and the tools correctly.

A specific experimental device allowing cutting conditions control and thin observations has been developed. This machine allows to measure strains during the cutting process through the use of gauges and KYOWA amplifier linked to an acquisition system LDS Nicollet. An acquisition frequency equal to $1 \mathrm{MHz}$ on each voices is available with a very limited noise which represents around less than $2 \%$ of the maximum signal. The speed of the punch is measured with a vibrometer-laser, used with a 250 $\mathrm{KHz}$ acquisition frequency. Moreover some industrial specificities are included on the experimental device such as a die and the possibility of controlling the clearance between die and punch, around $0,05 \mathrm{~mm}$ ie less than $2 \%$.

Marouani and al. studied the effect of the punch velocity and the clearance on the cutting force in the case of a FeSi steel [3]. They showed that the maximum punch force is nearly not affected by one of those parameters. However the maximum punch speed imposed, is $123 \mathrm{~mm} / \mathrm{s}: 10$ times less than what that is expected in high speed blanking. Ong and Chan have reported that for different materials (mild steel, stainless, aluminium, brass and copper), the peak load is higher in high speed blanking

a e-mail: camille.gaudilliere@ensam.eu 
than for conventional speed [4]. However the energy used during the high speed process seems to be around $10 \%$ less than what it is used for low speed process. According to [1], the temperature increases in a very located area (around $10 \mu \mathrm{m}$ and in a very short time, less than $100 \mu \mathrm{s}$ ), provocates adiabatic shearing bands. This phenomena may reduce peak loading or energy needed to cut the sheet metal. However, to observe adiabatic shearing bands the termal conductivity of the material should be low. So, some material like steel C40 presenting a $51 W \cdot M^{-} 1 . K^{-} 1$ thermal conductivity should be a good candidate.

Consequently, according to those remarks, this study is interested by obtaining the cutting evolution involved during the C40 steel cutting for differents punch speeds since 5 up to $20 \mathrm{~m} / \mathrm{s}$. The cutting force evaluation can only be done if the device characteristics are taken into account. So, the first part of the paper presents the experimental device. In the second part of the paper, the calibration of the experimental device is detailled. Differents shots are then made in order to calculate cutting forces with a new method. Finally, some microscopic observations are done to understand if the evolution of cutting forces is related to the presence of adiabatic shearing bands.

\section{Experimental device}

The experimental device developped in our laboratory, is composed of three differents zones, (fig.1) [5].

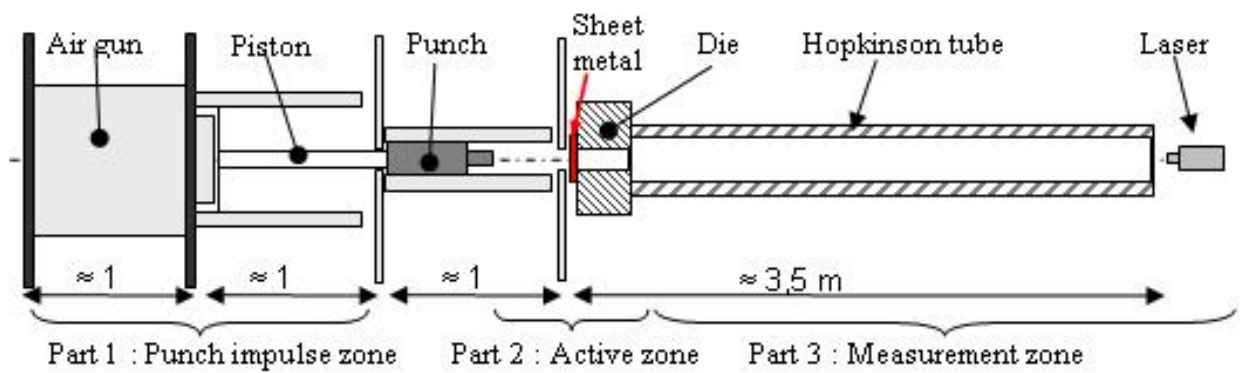

Fig. 1. Experimental device

The first zone, called the punch impulse zone, is composed by an air system gun. The shot is provocated after control of the pressure in the tank. The punch speed can be controlled very precisely from 5 up to $20 \mathrm{~m} / \mathrm{s}$. Two guidances drive the punch to the specimen with a clearance less than $1 \mu \mathrm{m}$. The second part of the device, called the active zone, is composed by the specimen and the die. The third zone, called the measurement zone, is for sensors and more specifically for gauges. The die is linked to the Hopkinson tube by a thread assembly. When the punch touches the specimen, a wave is generated and is transmitted to the tube via the die. Gauges are glued on the tube in order to measure strains. The laser measures the punch velocity during the shot with a precision of less than $0.1 \mathrm{~m} / \mathrm{s}$.

The die, its thread assembly with the tube and its complex 3D geometry, change the impedance of the tube. As a consequence, the elastic wave theory could not be used. Cutting forces have to be calculated by an other method.

\section{Cutting force calculation}

An other technique is presented, allowing the calculation of shear forces and taking into account of the industrial configuration of the proposed test. 


\subsection{Experimental device for the calibration step of elementary signals}

The main difficulty is to apply a force which can be known and controlled, as it can be done during the cutting process. The time of the cutting solicitation is around $200 \mu \mathrm{s}$. As a consequence, the best choice is to use another Hopkinson apparatus (fig.2)[6], [7]. An instrumented bar, the input bar, with gauges is put on one side, on the active surface of the die (fig.2). On the other side of the input bar, an air-gun system has been designed to launch projectiles. The forces imposed by the projectile to the die are measured from the gauges signals on the input bar.

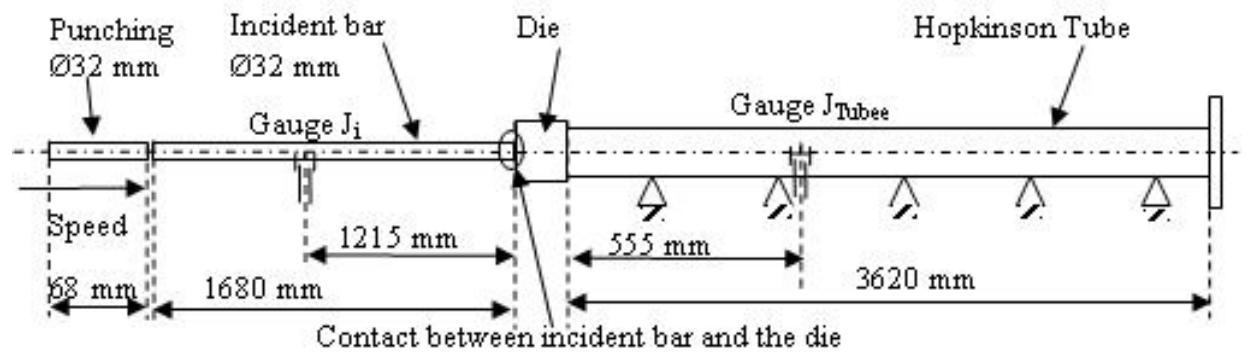

Fig. 2. The experimental device for the calibration

First, the relation between the strain gauge and the cutting force is supposed linear and can be justify experimentally. Consequently, if we apply input forces $F_{i}(t)$, which amplitude are close to the amplitude of the forces obtained during the cutting process, and measure the corresponding strains $\epsilon_{i}(t)$, we can reconstruct cutting forces $F_{\text {calc }}(t)$ by comparison of strains mesured during the cutting process $\epsilon_{m}(t)$ and the strains obtained during the calibration $\epsilon_{i}(t)$.

When the projectile touches the incident bar, a compressive wave is created in the calibration bar. This wave, the incident wave $\left(\epsilon_{\text {incident }}\right)$, propagates into the incident bar up to the die. On this interface the wave is split into a reflected $\left(\epsilon_{\text {reflected }}\right)$ and a transmitted wave which is measured with the gauge Jtube (fig.2). The strains induced by this wave, enables us to estimate the applied forces on the die. The forces applied on the die can be evaluated considering the waves gauges measurements and the elastic wave theory (eq.1):

$$
F=E S\left(\epsilon_{\text {incident }}+\epsilon_{\text {reflected }}\right)
$$

$\mathrm{E}$ is the Young modulus (206 GPa) and $\mathrm{S}$ is the section of the incident bar $\left(804 \mathrm{~mm}^{2}\right)$.

From this setup, the output signals measured from strain gauges located on the tube can be associated with the different input force signals.
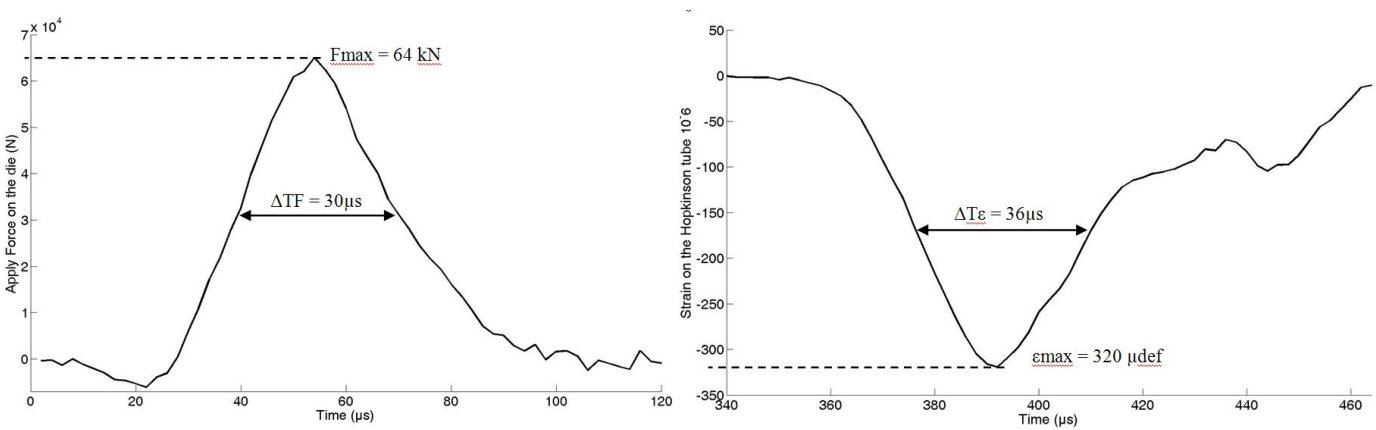

Fig. 3. Forces at the interface bar/die and strains on the Hopkinson tube

The figure 3 shows the elementary signals used for calibration. 


\subsection{Calculation process for the reconstruction of cutting forces}

The cutting forces have to be built from the elementary measured signals. However, the strain measured during the cutting process, $\epsilon_{m}(t)$, is quite different to the strain obtained during the calibration $\epsilon_{i}(t)$. The strain signal $\epsilon_{i}(t)$ has to be enriched with the addition of elementary signals to have the best approximation of the cutting force. Moreover, the relation between the strain gauge and the cutting force is supposed linear. Furthermore, a temporal lag of the force generates the same temporal lag on the strain measured on the tube. Consequently, families of curves of calibration can be easily obtained: either by time-shifting curves of the initial couple or either by multiplying with a coefficient those curves.

Each family has been built by translating the first element from a time-gap, which is optimized to have a good precision in our representation and at the same time to have a time calculation as little as possible.

The figure 4 represents the measured strains on the tube during the cutting process and the elementary signals needed to built the strains. The time-gap between each signals is $12 \mu \mathrm{s}$.

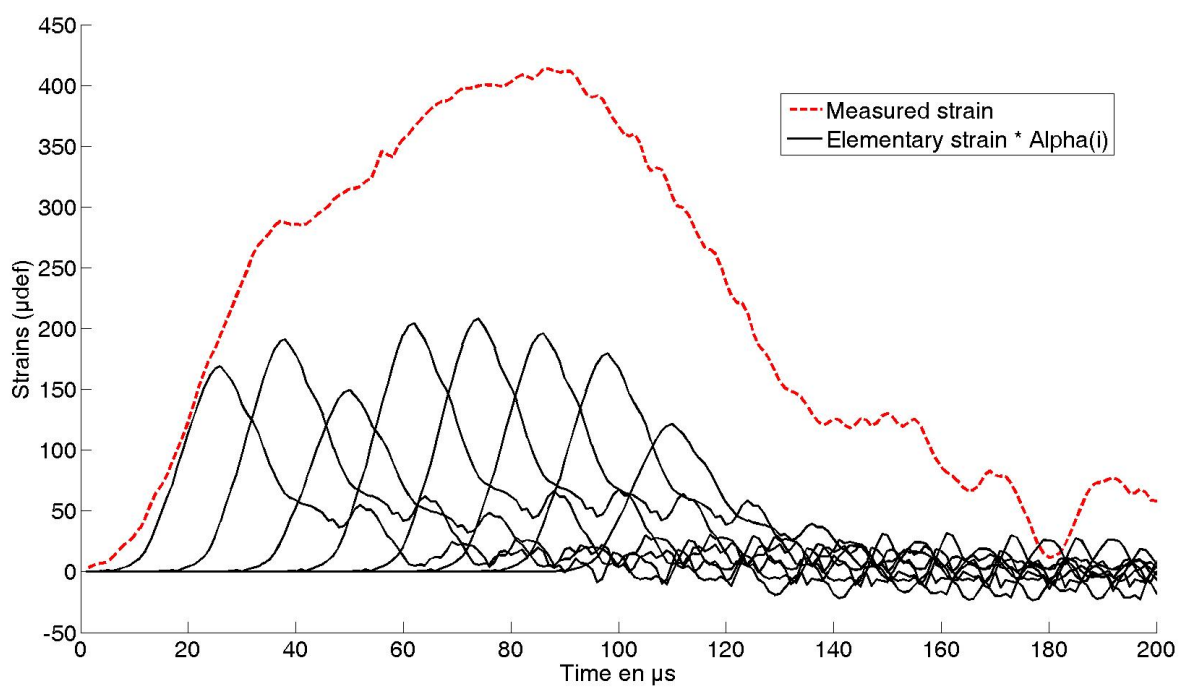

Fig. 4. Measured strains and elementary signals needed for the calculation

As a consequence, the measured strain $\left(\epsilon_{m}(t)\right)$ during the cutting process could be represented by a combination of elements $\left(\epsilon_{i}(t)\right)$ of the strains family :

$$
\epsilon_{m}(t)=\sum\left(\alpha_{i} * \epsilon_{i}(t)\right)+r(t)
$$

$\mathrm{r}(\mathrm{t})$ is the gap between the measure of the wave induced by the punch impact on the specimen, and the linear combination of signals from the calibration. The method is based on the minimisation of the gap $r(t)$.

The equation 3 lets us calculate $F_{\text {calc }}(t)$ :

$$
F_{\text {calc }}(t)=\sum\left(\alpha_{i} * F_{i}(t)\right)
$$

The reconstruction method has been validated through the use of a numerical simulation, which reproduces a dynamic behaviour similar to the dynamic of the experimental device. In fact we just 
want to verify that, the minimization of $\mathrm{r}(\mathrm{t})$ induces the minimization of the gap between $F_{m}(t)$, the real cutting force and $F_{\text {calc }}(t)$.

Different shots at different speeds, enable the calculation of the cutting forces using the preceding method. This force calculation is compared with the force obtained using the elastic wave theory. By using this second technique, even if it seems to be more simple because of it does not need a calibration, the presence of the die is neglected. The figure 5 shows the cutting forces calculated by the two methods. The thickness specimen is $3 \mathrm{~mm}+/-0,1 \mathrm{~mm}$ and the punch speed is around $13.9 \mathrm{~m} / \mathrm{s}+/-$ $0,1 \mathrm{~m} / \mathrm{s}$. The clearance between punch and die is about $0,05 \mathrm{~mm}+/-0,001 \mathrm{~mm}$.

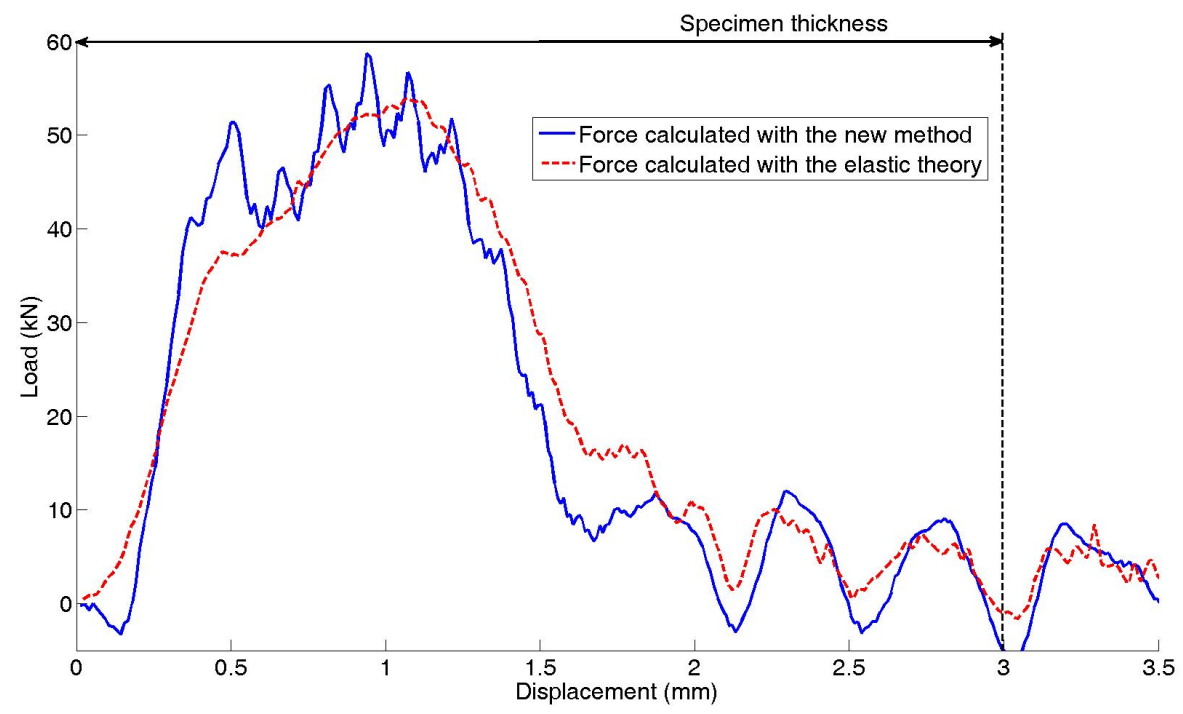

Fig. 5. Cutting force versus punch displacement: steel $\mathrm{C} 40$ and punch speed $=13.9 \mathrm{~m} / \mathrm{s}$

The gap between the two curves is very small after $1 \mathrm{~mm}$ displacement of the punch. However, a strong difference appears at the beginning: our calculation method gives a first peak load which has been observed by Ong and Chan [4]. The small negative force could be explained considering that the tube is puched when the punch touches the sheet metal. Just after, a strain localization occurs and the first peack can then be observed. Indeed, a shear band is created and some friction appears between each part of the specimen. Finally, the sheet is cut because of the shear band propagation all over the specimen thickness. Consequently, when the sheet of metal is sheared, the force decreases very rapidly. Finally, the peack load appears at the same time whatever the calculation method.

Moreover, fig. 5 shows that the sheet is cut when the displacement of the punch raises $2 \mathrm{~mm}$. The Hopkinson tube which supported the die and the specimen is pushed and the workpiece is a small bent, so the punch penetration on the sheet metal is less than $2 / 3$ of the thickness of that one. This phenomena has already been observed for a $11.6 \mathrm{~m} / \mathrm{s}$ speed [4].

The validation of that new calculation method is done and enables to obtain the cutting forces. The test bench enables now to analyse the influence of the punch speed on the cutting forces history and its influence on the peak load. 
Differents tests have been made: specimens of steel C40,3mm thickness, have been cut for speed between 10.6 and $18.1 \mathrm{~m} / \mathrm{s}$. The clearance between the die and the punch is $0.05 \mathrm{~mm}$, and the weight of the punch is around $1.5 \mathrm{~kg}+/-0.03 \mathrm{~kg}$.

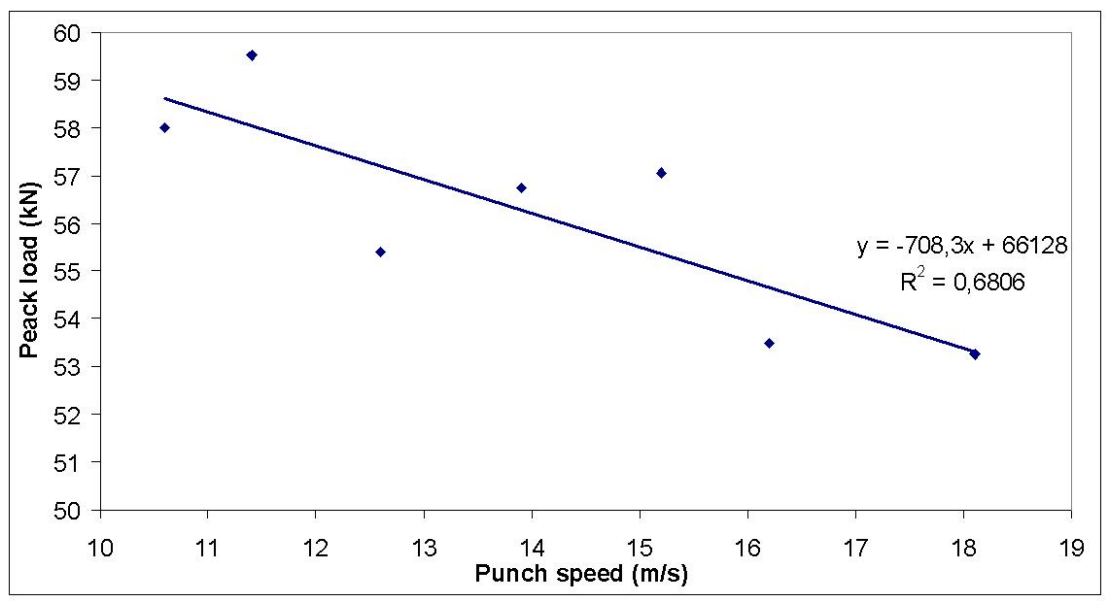

Fig. 6. Evolution of the peack load versus the punch speed: steel C40

The measurement uncertainty is estimated around $2 \%$, which can explain the important difference between each value. However, fig.6 shows that the peack load decreases when the punch speed increases.

What evolution does the feed generate in the metal structure in order to get such an evolution of the cutting force peak? To try to explain, some investigations concerning the fracture facies have been made.

\section{Microscopic observations}

Each test specimen is cut in three parts. First part corresponds to the middle of the specimen, where the punch is in contact, and the two other parts correspond to the contact between die and sheet metal. First observations are made on the middle part at the middle of the workpiece height.

The observation of the cutting blanks with an optic microscope enables to find adiabatic shearing bands (ASB) 7 .

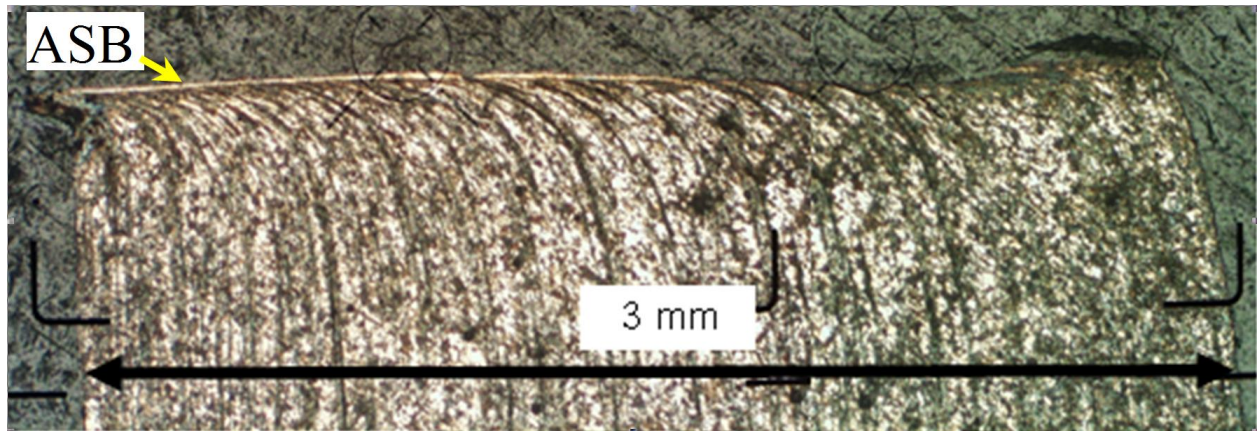

Fig. 7. Adiabatic Shearing Band: C40, 3mm thickness, Punch speed $=16,2 \mathrm{~m} / \mathrm{s}$ 
Those ASB are observed on the two cutting faces and on the differents parts of the specimen. When the speed is too low, no ASB can be observed. Those observations help us to conclude that the evolution of the cutting force versus speed might be partly explained by the creation of ASB.

\section{Conclusion}

The experimental method has been successfully implemented for the determination of shear forces induced by the cutting of a steel C40 part. The effect of the cutting speed variation on the shear forces are presented and discussed. Some observations are made in order to understand how the sheet of metal is cut. Microscopics observations show that at high punch speed the presence of ASB is clearly identified. This study helps us to suppose that ASB are playing a role in the decreasing process of the peack forces.

\section{References}

1. L. Lazzarotto, R. Michon, 5th Car Body Colloquium,(2008) 13

2. S. Jana, N.S. Ong, Journal of Mechanical Working Technology 19,(1989) 17

3. H. Marouani, A. Ben Ismail, E. Hug, M. Rachik, Materials \& Design, 30,(2009) 5

4. N.S. Ong, L.T. Chan, Journal of Mechanical Working Technology 18,(1989) 14

5. C. Gaudilliere, N. Ranc, A. Larue, P. Lorong, 9th International conference on the mechanical and physical bahaviour of materials under dynamic loading 1,(2009) 6

6. P.S. Follansbee, C. Frantz, Journal of Engineering Materials and Technology, Transactions of the ASME 105,(1983) 5

7. H. Zhao, Computers and Structures 81,(2003) 9 